\title{
Les villes au piège de la précarité Généalogie d'un souci des usages fragiles de l'espace urbain
}

\author{
Christophe Mager, Lausanne, Laurent Matthey, \\ Genève
}

\section{Introduction}

Dans un bel optimisme, typique des Trente Glorieuses, Robert Nisbet faisait, en 1959, l'hypothèse d'une fin des classes sociales. La diffusion de la propriété dans toutes les couches de la société rendait obsolètes les anciennes différenciations sociétales. L'élévation du niveau de vie conduisait à l'affaiblissement des frontières sociales en terme d'accès à la consommation, aux références culturelles et à la mobilité (PAKULSKI \& WATERS 1996). Il semble toutefois que tant l'histoire économique que l'histoire sociale des sociétés occidentales se soient liguées pour donner tort au sociologue conservateur. Le monde social général persiste à être structuré en groupes hiérarchiques distincts, repérables, identifiés et opposés. Bien plus, l'extension de la classe moyenne n'a pas résolu les problèmes de pauvreté. Cette double défaite de la prophétie nisbetienne se traduit dans la morphologie des espaces urbains, qui devient un agent des processus de différenciation en même temps qu'elle assure l'inertie de la morphologie sociale; questionnant par là même l'efficacité des politiques publiques en matière de gestion des usages fragiles de la ville.

Cette contribution aspire à discuter les différentes explications du délitement du contrat social tel qu'il était rêvé par Robert Nisbet. Questionnant ce qu'il est convenu d'appeler la montée de la précarité dans les centres urbains, elle détaille dans un premier temps les mécanismes économiques, institutionnels et les effets de lieux qui la fondent. Dans un deuxième temps, ce sont les modalités émergentes d'accompagnement des usages fragiles de la ville - à savoir les pratiques urbaines des usagers les moins légitimés par l'ordre normatif incarné par la ville ( $\mathrm{p}$. ex. toxicomanes, personnes vivant dans la pauvreté ou sur sa frontière, sans-papiers et illégaux, migrants de première génération) - qui sont explicitées. Pour ce faire, deux types de sources sont mobilisés: des données et conclusions tirées de la littérature spécifique; des données d'enquêtes réalisées depuis 2004 à l'Observatoire de la ville et du développement durable de l'Université de Lausanne. L'exercice proposé est donc celui d'un état de l'art réflexif et critique.

\section{Une montée de la précarité? Nouveaux régimes d'accumulation, double détente des politiques publiques et piège du tri urbain}

En dépit des vœux pieux de Robert Nisbet, la tension fondamentale animant l'espace social ne s'est pas résolue dans une généralisation de la classe moyenne, pas plus que cette extension n'a fait disparaître la pauvreté, invalidant, une fois de plus, la courbe de SIMON KuZNETs (1955). Sous l'effet des politiques sociales, singulièrement sous leur aspect assurantiel (RosANvaLLON 1981), les formes de pauvreté urbaine ont toutefois changé. Par exemple, si les retraités ont longtemps constitué la plus grande partie des pauvres résidant en ville, ceux-ci ont vu leur niveau de vie moyen progresser. En revanche, la dégradation de la condition salariale et la progression des emplois précaires sont à l'origine de la réapparition d'une forme de pauvreté métropolitaine.

Trois éléments peuvent être convoqués pour expliquer ce mouvement. Le premier relève de ce que l'école régulationniste a appelé une mutation du régime d'accumulation. Le deuxième met l'emphase sur les conséquences imprévues des politiques publiques en matière de lutte contre le chômage ou de réinsertion socio-professionnelle. Le troisième fait référence aux effets de tri et d'inertie des territoires urbains.

\subsection{Les effets de recomposition du régime d'accumulation}

Le premier élément susceptible d'expliquer l'échec du pari nisbetien consiste très certainement dans cette mutation du régime d'accumulation capitalistique qu'on a coutume d'appeler la mondialisation productive. En faisant de la flexibilisation de la main-d'œuvre l'une des conditions essentielles d'adaptation des entreprises à leur mise en concurrence croissante, cette mondialisation a en effet contribué à la reproduction des frontières sociales. Il s'agit toutefois d'être clair quant au contenu de la flexibilité. Appliquée au processus de production, la flexibilité affecte la relation d'emploi qui désigne, selon la définition qu'en donnent LAURENT TASKIn \& Marie Schots (2005), non seulement un mode de construction sociale du salariat en tant que sujet politique collectif et agent économique inséré dans des relations d'échange, mais aussi une codification juridico-contractuelle. Partant, la flexibilisation de la relation d'emploi affecte le système de rémunération, la construction des qualifications et l'orientation des mobilités professionnelles (BARDET et al. 2010). 
En ce qui concerne les rémunérations, les entreprises cherchent à transformer la masse salariale de coût fixe en coût variable, c'est-à-dire à l'ajuster aux variations de la production (ORGANISATION DE COOPÉRATION ET DE DÉvELOPPEMENT ÉCONOMIQUES - OCDE 2004). Pour ce faire, les employeurs tendent à flexibiliser les coûts salariaux en minimisant la part fixe des salaires, une part croissante de la rémunération étant à la tâche, au mérite ou liée aux résultats des entreprises, induisant des formes d'engagement qui ne procurent aucune garantie quant au montant du salaire. Cette flexibilisation s'articule à une augmentation relative des contrats à durée déterminée. Toutes choses participant à la création d'une main-d'œuvre flottante, dont la protection sociale est réduite ou inexistante. Cette main-d'œuvre flottante compose une large part des working poor, c'est-à-dire la proportion de pauvres parmi les personnes qui exercent une activité professionnelle. De fait, la pauvreté en Suisse est certes marginale, mais elle n'est pas négligeable. Le taux de working poor en 2007 y était de $4,4 \%$ (7,4\% en 2003$)$, le taux de pauvreté des 20-59 ans atteignant lui 8,8\% (OFFICE FÉdÉRAL DE LA STATISTIQUE - OFS 2006, 2009a, 2009b). Quant au taux de bénéficiaires de l'aide sociale par rapport à la population, il a été en moyenne d'environ $3 \%$ entre 2005 et 2008 (OFS 2008). La part des personnes engagées à temps partiel a, quant à elle, nettement progressé depuis les années 2000 . Leur nombre a augmenté de 118500 unités $(+10,6 \%)$ de 2005 à 2008 . Alors qu'en 1998, un quart seulement de la population active occupée travaillait à temps partiel, cette proportion se montait déjà à 30\% en 2005 (OFS 2010). Pour ces personnes aux faibles capitaux, le logement et l'habiter relèvent plus de l'assignation résidentielle que du choix (Fitoussi et al. 2004).

Le nivellement par le bas des fondements du rapport salarial institué par le régime d'accumulation fordiste n'a toutefois pas été généralisé. Si les mutations du modèle productif tendent à précariser les moins qualifiés du fait de la nécessité d'une flexibilité dans les processus, elles exigent également une protection du personnel le plus qualifié (BARDET et al.2010). Ce dernier a donc un pouvoir de négociation salarial important, d'autant plus fort qu'il dispose de compétences transversales le rendant davantage apte à innover, à anticiper et à réagir rapidement aux fluctuations de la demande et aux éventuelles difficultés pouvant surgir dans le processus de production. Nombre d'entreprises mènent ainsi, conjointement à une flexibilisation, une politique de valorisation des ressources en formant leur personnel qualifié, en se préoccupant de lui fournir les opportunités d'une carrière, en stimulant sa créativité et en conservant ces employés même quand les affaires vont un peu plus mal, afin de s'assurer de leur loyauté et de leur engagement (BÉRUBÉ 2006).
En théorie, les inégalités sociales induites sont souvent évoquées comme la condition sine qua non de la croissance économique urbaine, la prospérité des riches étant considérée comme un puissant soutien à l'investissement productif et à la création d'emplois de tous types (LANDAIs 2007). En réalité, la reproduction de ces inégalités a un coût dont il serait hasardeux d'affirmer qu'il est inférieur aux bénéfices sociétaux espérés (CleRC 2010).

\subsection{L'effet paradoxal des politiques publiques}

La «déstabilisation des stables» (CASTEL 1995) dans laquelle s'inscrit la montée de la précarité n'est pas du seul ressort d'un certain mode d'accumulation capitalistique; elle relève également d'un certain mode de gestion politique des cycles économiques. À titre d'exemple, le système suisse d'indemnisation du chômage ne s'est guère adapté aux mutations du marché $\mathrm{du}$ travail. Les nouveaux risques liés aux fonctionnements présents du marché du travail ne sont pas ou sont mal pris en compte: instabilité croissante des emplois et des carrières qui précèdent ou entrecoupent les périodes de chômage, développement des emplois précaires, allongement des processus d'insertion des jeunes. De plus, les politiques sociales organisent un cumul sans précédent d'incitations monétaires et non monétaires, du côté de l'offre comme de la demande qui, en combinant des aides ponctuelles, des subventions temporaires et des aides permanentes, compliquent la lisibilité de l'État-providence, contribuant même, parfois, à construire ce que d'aucuns appellent les «carrières des pauvres» (Paugam \& Duvoux 2008) en enfermant - selon une logique déjà identifiée par Simmel (1908 [2005]) - les plus démunis dans des dispositifs qui les fragilisent.

Ainsi, il a été montré (Paugam \& Duvoux 2008) que la précarité résultait de deux processus qui, d'origine distincte, convergeaient dans leurs effets: celui d'une politique de flexibilisation de la main-d'œuvre, qui relève d'une logique entrepreneuriale; celui d'une politique de l'emploi qui vise notamment à réinsérer des personnes peu qualifiées et à offrir des opportunités de formation aux jeunes. La politique des stages et de mise à l'emploi précoce, justifiée par une nécessité d'expérience à acquérir, produit de la vulnérabilité, de sorte qu'on a ici affaire à des dispositifs d'activation et de régulation qui concourent paradoxalement à une fragilisation des statuts (PAugam 1991; PAugam \& Duvoux 2008). Au final, la convergence de ces deux processus produit une stratification sociale du salariat, particulièrement préoccupante dans les villes-centres, d'autant qu'elle susciterait l'émergence d'une nouvelle figure sociétale, celle du «travailleur précaire assisté» (PAUGAM \& MARTIN 2009), mis au travail dans les secteurs les plus dégradés (bad jobs) de l'économie. 
Le plus préoccupant consiste sans doute dans ce que cette déstabilisation des stables conduit à ce que Nicolas Duvoux et Serge Paugam (2008) ont identifié comme une «usure de la compassion». Graduellement, les personnes en situation de fragilité tendent à être identifiées à des «fraudeurs». En effet, les gains d'un working poor sont parfois très proches de ceux d'un allocataire de prestations sociales, ce qui occasionne, au terme d'une certaine forme de comparaison envieuse, un ressentiment. L'ironie consiste sans doute dans ce que ce ressentiment, organisé politiquement, concourt à la mise en place de dispositifs d'activation qui visent à remettre au travail des allocataires, fragilisant, au second tour, une nouvelle fois les précaires.

En somme, il n'exis te visiblement pas, à l'heure actuelle, de pratiques visant à la diminution des fragilités urbaines dont on pourrait s'inspirer sans risque d'effets pervers ou d'efficacité limitée (MAGer \& Stofer 2009). Pis même, certaines de ces politiques contribuent, au moins en partie, à la reproduction des inégalités sociospatiales en étant incapables de contrer à large échelle les forces du marché, amenant les pouvoirs publics à passer, selon l'expression de STÉPHANE CADIOU (2006), du volontarisme à la perplexité.

\subsection{L'effet d'inertie des territoires}

Enfin, la montée de la précarité dans les villes-centres est un effet collatéral de ce qu'il est convenu d'appeler le tri urbain. La couronne suburbaine filtre les ménages non qualifiés, les ouvriers et les employés, arrêtés dans leur aspiration à la périurbanité. Leur solvabilité déficiente les exclut de facto de l'accès aux crédits hypothécaires. Captifs des espaces urbains centraux, il n'y a guère d'effort à faire pour les y maintenir. Ce qui ne signifie pourtant pas qu'il soit inutile de faire en sorte qu'ils puissent disposer d'une offre de logements et de services répondant à la fois à leurs attentes, à leur budget et à la perspective d'une bonne qualité de vie urbaine (CunHA et al.2007).

L'étalement urbain a donc amplifié la propension des classes à se démarquer dans l'espace (CunHA 2007), s'inscrivant dans une logique qui est proprement celle de la ségrégation spatiale (voir tab. 1): les «dirigeants» et les «professions libérales» ont propension à se concentrer dans les zones périurbaines, de même pour les «professions intellectuelles et d'encadrement» et les «autres indépendants» (CunHA \& BoTH 2004). A contrario, les «ouvriers» et les «travailleurs non qualifiés» se retrouvent dans les communes centrales et les zones suburbaines (CUNHA \& BoTH 2004). On observe à l'échelle des cinq agglomérations suisses un phénomène de polarisation sociale de l'espace déjà documenté par THÉRÈse Huissoud et al. (1999). Cette polarisation s'explique notamment par la structure du marché foncier, l'offre de logements, les aspirations résidentielles et la capacité financière des ménages. Trois dimensions qui font système et convergent pour inscrire durablement les fragilités dans les villescentres qui pâtissent ainsi du fait que le capitalisme a renoué avec ses tendances - inscrite dans le temps long - à la paupérisation des plus faibles, mais aussi à l'accentuation des inégalités.

Bien entendu, les dynamiques urbaines observées ne sont pas seulement le résultat d'un tri par le marché foncier, mais sont également imputables à une «préférence» pour un entre-soi, que l'on sait s'alimenter à divers critères tels que le niveau de richesse, les pratiques religieuses, l'appartenance ethnique (ZHAo et al. 2006) ou encore être induit par des mécanismes autoentretenus aboutissant à une hystérésis importante dans les zones de relégation (THIsse 2004). L'inertie est d'autant plus forte que les indices d'une gentrification émergente des villes suisses ne résolvent pas la tension née d'une forte dualisation des espaces centraux.

Pour rappel, la gentrification fait référence à un retour en ville d'habitants - souvent fortement dotés en capital économique, culturel et social - qui avaient choisi de s'en exiler à un moment de leur parcours biographique (Donzelot 2004). Les données du Recensement fédéral de la population 2000 inclinent ici à un usage plus prudent du terme en ce qui concerne les cinq grandes agglomérations du pays (voir tab. 2). En effet, la croissance démographique des villescentres est bien moins la conséquence d'un retour en ville des «exilés» du périurbain que de mouvements interurbains de centre à centre, de regroupements familiaux ou d'une nouvelle migration internationale. La part $\mathrm{du}$ «retour en ville» dans la croissance démographique des centres s'élève à $8 \%$ tout au plus. Ainsi, la structure sociale des villes n'a pratiquement pas changé en trente ans. Les centres concentrent les ménages célibataires, les populations étrangères, les personnes âgées. Bien plus qu'une gentrification, on observe ainsi, dans les villes-centres, un «émiettement du tissu social» (CUNHA \& Bотн 2004) caractérisé par une montée des ménages isolés, un vieillissement de la population encore renforcé par le retour de quelques seniors pionniers du périurbain (RÉRAT et al. 2008) et partiellement atténué par l'arrivée de jeunes adultes en formation.

En outre, une part importante de la croissance démographique des centres-villes est liée aux migrations internationales. Les cinq principales villes du pays captent environ $25 \%$ des flux de personnes en provenance de l'étranger. Les données du dernier recensement montrent par ailleurs que sur les 194686 personnes récemment établies dans les communes centrales, $36 \%$ viennent d'un pays étranger. De fait, l'établissement des migrants est déterminé à la fois par les 


\begin{tabular}{|c|c|c|c|c|c|}
\hline \multirow{2}{*}{$\begin{array}{l}\text { Catégorie socio- } \\
\text { professionnelle }\end{array}$} & \multirow{2}{*}{$\begin{array}{l}\text { Répartition en } \\
\text { Suisse en \% }\end{array}$} & $\begin{array}{l}\text { Villes- } \\
\text { centres }\end{array}$ & $\begin{array}{l}\text { Autres } \\
\text { communes } \\
\text { centrales }\end{array}$ & $\begin{array}{l}\text { Communes } \\
\text { suburbaines }\end{array}$ & $\begin{array}{l}\text { Communes } \\
\text { périurbaines }\end{array}$ \\
\hline & & \multicolumn{4}{|c|}{ Indices de localisation } \\
\hline Dirigeants & 3,4 & 0,92 & 0,99 & 0,94 & 1,21 \\
\hline Professions libérales & 2,1 & 1,21 & 0,68 & 0,69 & 1,37 \\
\hline Autres indépendants & 10,2 & 0,87 & 0,92 & 1,01 & 1,30 \\
\hline $\begin{array}{l}\text { Professions } \\
\text { intellectuelles et } \\
\text { d'encadrement }\end{array}$ & 14,5 & 1,20 & 0,79 & 0,80 & 1,13 \\
\hline $\begin{array}{l}\text { Professions } \\
\text { intermédiaires }\end{array}$ & 22,2 & 0,98 & 0,99 & 1,02 & 1,03 \\
\hline $\begin{array}{l}\text { Non-manuels } \\
\text { qualifiées: employés }\end{array}$ & 26,3 & 0,96 & 1,06 & 1,07 & 0,91 \\
\hline $\begin{array}{l}\text { Manuels qualifiés: } \\
\text { ouvriers }\end{array}$ & 7,4 & 0,82 & 1,15 & 1,16 & 0,94 \\
\hline $\begin{array}{l}\text { Travailleurs non } \\
\text { qualifiées }\end{array}$ & 13,9 & 1,08 & 1,14 & 1,01 & 0,69 \\
\hline
\end{tabular}

Tab. 1: Population active occupée des grandes agglomérations, selon les catégories socio-professionnelles et le type de zone (indice de localisation), en 2000

Number of employed persons in large agglomerations in 2000, according to socio-professional categories and zone types (locality index)

Aktiv beschäftigte Bevölkerung der grossen Agglomerationen, gemäss den sozio-professionellen Kategorien und dem Zonentyp (Lokalisationsindex), im Jahr 2000

Source: CunHA \& BOTH 2004: 72

opportunités de travail, de logement et l'existence de réseaux d'interconnaissance. Or, c'est principalement dans les centres-villes que l'on trouve le plus souvent des emplois peu qualifiés, singulièrement dans le secteur des services. Par ailleurs, il y existe une offre de ce que antonio Da Cunha \& Jean-François Both (2004: 89) appellent le «logement social de fait», à savoir des logements peu onéreux, «parfois anciens et moins confortables» sinon insalubres. Enfin, les villes-centres connaissent une concentration préalable (CunHA et al. 2003) de population migrante qui a pour effet de polariser les flux.

Les Accords bilatéraux entre la Suisse et l'Union européenne contribuent néanmoins à transformer ce modèle puisqu'une main-d'œuvre étrangère beaucoup plus qualifiée collabore à présent à la croissance démographique des espaces urbains, participant à sa manière à la dualisation des centres. Cette population plus qualifiée occupe en effet des segments plus favorables d'un marché immobilier lui-même très ségrégé. Il se pourrait en outre que la gentrification des espaces urbains, que certains observent à titre de frémissement, soit en partie imputable à l'arrivée, dans les centres-villes, de cette nouvelle migration, mieux formée, aux capitaux sociaux et culturels plus élevés (MEYER 2008).

\section{Un retour de la question urbaine}

Cette explication multidimensionnelle de l'augmentation de la précarité dans les centres-villes suisses 


\begin{tabular}{|l|rcc|cc|}
\hline & \multicolumn{3}{|c|}{ Flux entrants } & \multicolumn{2}{c|}{ Flux sortants } \\
\cline { 2 - 6 } $\begin{array}{l}\text { Types de zones de } \\
\text { provenance }\end{array}$ & Effectifs & En \% du total & $\begin{array}{c}\text { En \% des migrants } \\
\text { internes }\end{array}$ & Effectifs & $\begin{array}{l}\text { En \% des migrants } \\
\text { internes }\end{array}$ \\
\hline $\begin{array}{l}\text { Même } \\
\text { agglomération }\end{array}$ & 57292 & 29,4 & 46,0 & 84944 & 64,4 \\
$\begin{array}{l}\text { Autre zone } \\
\text { urbaine }\end{array}$ & 46692 & 24,0 & 37,5 & 31650 & 24,0 \\
$\begin{array}{l}\text { Communes rurales } \\
\begin{array}{l}\text { Migrations } \\
\text { internes }\end{array}\end{array}$ & 20656 & 10,6 & 16,6 & 15383 & 11,7 \\
$\begin{array}{l}\text { Pays étranger } \\
\text { Migrations totales }\end{array}$ & 124640 & 64,0 & 100,0 & 131977 & 100,0 \\
\hline
\end{tabular}

(Note: Les pourcentages ont été arrondis à la décimale supérieure; N.D.* $=$ non disponible)

Tab. 2: Flux de migrants des villes-centres des grandes agglomérations, selon le type de zones de provenance, en 1995/2000

Migration flux from urban centres of large agglomerations in 1995/2000, according to categories of origin Wanderungsströme aus den Stadtzentren der grossen Agglomerationen, gemäss dem Typ der Herkunftszonen, in den Jahren 1995/2000

Source: CUNHA \& Both 2004: 75

conduit à reformuler ce que JACQUES DonZeLot (2006) a nommé la «question urbaine».

\subsection{Les politiques urbaines à l'heure du délitement de la société salariale}

Pour JaCQues Donzelot (2004), la question sociale telle qu'elle a été thématisée au XIX ${ }^{e}$ siècle a trouvé sa résolution dans l'élucidation de la question urbaine qui en constituait le calque en même temps que l'analyseur. Le compromis élaboré consistait à éloigner les «classes dangereuses» - pour reprendre l'expression de Louis CHEVALIER (1958), devenue proverbiale - tout en maintenant la possibilité d'une mobilité sociale, lisible dans la continuité de la ville:

«la ville moderne, la ville de l'industrialisation réussi[t] à <faire société> par le tracé fonctionnel de son espace, la mise à distance des classes antagonistes et les pointillés du rêve de la promotion sociale individuelle» (Donzelot 2004: 15).

La montée de la précarité dans certaines poches des espaces centraux (près du quart des 234000 personnes à l'aide sociale en Suisse réside dans les cinq principales villes du pays, selon les données de 2007), et l'immobilité qui caractérise les personnes qui en sont frappées (au sens où leur choix résidentiels sont contraints) conduisent à s'interroger sur les issues possibles de la question urbaine telle qu'elle se pose en ce début de XXIe siècle. D'autant que les quelques opérations de requalification des centres, souvent lourdement médiatisées, ne suffiront pas à résoudre cette nouvelle tension.
Dans ce contexte, les villes ont fait le choix de travailler principalement sur deux axes qui témoignent de leur difficulté à administrer des logiques qui excédent parfois le périmètre de leur «sphère d'autorité» (Rosenau \& Singh 2002) - à l'exemple des dynamiques propres à l'économie mondialisée. Soit elles ont tenté de mimer le contrôle en développant des politiques de marketing urbain (MAGER \& MATTHEY 2010), soit elles se sont essayées à faire des usagers fragiles des acteurs de leur devenir.

\subsection{Entre empowerment et do it yourself: l'émergence d'un souci pour les usages fragiles de la ville}

Pour endiguer cette fragilisation et cette dualisation des espaces métropolitains, les municipalités développent actuellement - outre des politiques de déségrégation qui peuvent aller jusqu'à l'échelle de l'immeuble (AREND 2004) - des politiques visant soit à stimuler le retour dans les zones centrales de ménages à forte capacité contributive, soit à dissuader les ménages aisés de quitter les centres. Certaines communes réduisent le taux de logements subventionnés sur les terrains qu'elles mettent à disposition à des fins de construction et développent des projets de logements susceptibles de répondre aux aspirations résidentielles des exilés du périurbain. La mixité sociale se mue en un outil d'optimisation sociale du territoire (BONARD \& Matтhey 2010) en ce qu'elle permet une minimisation des inégalités (déconcentration des «problèmes» - difficultés scolaires, taux de chômage, faiblesses des revenus - et des «privilèges» - réussite scolaire, niveau élevé de revenus) et une maximisation du bien-être 
collectif (rééquilibrage géographique, que ce soit du point de vue du cadre de vie et de l'assiette fiscale des différents territoires).

Mais du point de vue de l'action publique, cet accroissement de la précarité se traduit plus significativement encore par l'émergence d'un souci marqué des usages fragiles de la ville. Depuis 1990, les dispositifs de lutte contre la pauvreté et la précarisation de l'emploi, les politiques d'intégration des populations migrantes, les opérations de prévention des violences ou de prévention des addictions constituent en effet des axes de structuration majeurs des agendas municipaux, stimulant l'invention de modalités nouvelles d'intervention et de gouvernance qui représentent certainement un point fort des développements sociaux qu'il est possible d'observer dans les villes suisses. La caractéristique commune de ces modalités nouvelles est d'adopter, autant que faire se peut, une conception «capabiliste» (SEN 2000) de la justice sociale, tant elle mobilise une rhétorique de l'empowerment, censé donner aux plus vulnérables la possibilité d'influer sur les politiques qui affectent leurs conditions de vie, en permettant de mieux identifier et prendre en compte leurs problèmes et leurs attentes.

Du point de vue des modalités de lutte contre l'exclusion, il est par exemple significatif que de nombreux dispositifs suisses d'aide à l'accès au logement ou de maintien dans le logement travaillent à rendre leurs habitants capables d'obtenir ou de conserver un bail (Cunha \& Schmid 2007a et b), que ce soit en assurant un suivi de la personne, en l'inscrivant dans un réseau, en lui permettant d'acquérir les compétences nécessaires à vivre en appartement, ou, enfin, en l'accompagnant dans la réduction de son endettement.

Cette conception de la justice sociale valorise souvent des outils qui relèvent de l'action communautaire ou de la mobilisation d'un «tiers secteur» (RACINE, MARENGO \& BLANC 2004) qui habilite ses membres en même temps que ceux-ci participent à la prise en charge de «problèmes». Ainsi, les quartiers sont désormais appelés à être une échelle pertinente de production des solidarités interpersonnelles ou intergénérationnelles. De même, les associations de migrants sont dorénavant convoquées à titre d'animateurs socioculturels dans certaines communes suisses (MATthey \& StEINER 2009a).

Par suite, les villes suisses jouent - dans le cadre de la nouvelle politique suisse d'intégration, caractérisée par un leadership étatique plus marqué - un rôle souvent novateur dans la tentative d'inventer des façons nouvelles de gouvernance, mais aussi de traduction des identités dans la migration; conduisant à ce qu'il s'y formule «des identités à la mesure du monde» (Matthey \& Steiner 2009b).
Les politiques de prévention des violences et de réduction du sentiment d'insécurité (RAcine \& NosedA 2005) tendent elles aussi à travailler à une plus grande emprise des habitants sur leur cadre de vie. Il convient désormais de faciliter la prise en charge de l'espace public par les résidents, que ce soit à l'occasion de budgets participatifs (comme par exemple dans la commune de Vernier dans le canton de Genève) ou de réaménagement d'échelles variables (du banc au quartier).

Il en va de même, enfin, en ce qui concerne les politiques et stratégies de prévention (et d'aide en cas de) des addictions. Les dispositifs élaborés au cœur des espaces métropolitains tendent à accroître les sentiments - et les possibilités effectives - de contrôle et de résilience des individus. Mais le projet est aussi de les réinscrire dans des réseaux d'entraide, dessinant l'ébauche d'un capital social susceptible d'être mobilisé pour accélérer la réinsertion des bénéficiaires (Cunha, Mager \& Schmid 2009).

\section{Repenser la citoyenneté urbaine}

La prophétie nisbetienne a difficilement supporté sa confrontation aux faits toujours têtus des recompositions économiques, des conséquences inattendues de nos politiques publiques et des effets d'inertie liés à la texture de nos territoires. Désemparées, cherchant à reprendre la main sur des phénomènes qui échappent au périmètre de leur sphère d'autorité, les villes développent des politiques qui mobilisent deux axes, l'un mimant le contrôle en développant des politiques de marketing urbain, l'autre jouant sur l'ambiguïté de la notion d'empowerment et du lien ténu qu'elle entretient avec le mode du do it yourself. Il reste que la question des vulnérabilités urbaines ne saurait se résoudre sans une reformulation du couple question sociale-question urbaine.

Plus fondamentalement, la question qui émerge ici est celle des modalités contemporaines de production d'une citoyenneté urbaine, qui dépasse les cadres usuels de l'action politique (le parti, le syndicat, l'association) pour assurer tout autant une égalité des chances et une égalité des places (Donzelot 2009).

\section{Bibliographie}

AREND, M. (2004): La planification urbaine et la politique du marché du logement peuvent-elles contribuer à une meilleures intégration des migrants? - In: WICKER, H.-R., Fibbi, R. \& W. Haug (éds): Migration und die Schweiz. Ergebnisse des Nationalen Forschungsprogramms «Migration und interkulturelle Beziehungen». - Zürich: Seismo Verlag: 227-244. 
Bardet, M., Blanchet, D. \& L. Crusson (2010): Globalisation et flux d'emploi. Que peut dire une approche comptable? - In: Economie et Statistique 427-428: 3-20.

BÉRubÉ, M.-E. (2006): La valorisation des ressources humaines en gestion stratégique des ressources humaines. Mémoire de maîtrise. - Montréal: École de relations industrielles, Université de Montréal.

Bonard, Y. \& L. Matthey (2010): Mixité n'est pas (mécaniquement) justice. La mixité sociale comme optimum de satisfaction sociétale? - In: Optimisation de l'espace géographique et satisfactions sociétales. - Avignon: Groupe Dupont et Université d'Avignon: 205-210.

CAdiou, S. (2006): L'état des segmentations. Les effets et dilemmes de l'activité gouvernementale. - Centre d'analyse stratégique. Rapport annuel 2006. - Paris: Imprimerie nationale: 133-144.

CASTEL, R. (1995): Les métamorphoses de la question sociale. - Paris: Fayard.

Chevalier, L. (1958): Classes laborieuses et classes dangereuses à Paris pendant la première moitié du $\mathrm{XIX}^{\mathrm{e}}$ siècle. - Paris: Plon.

Clere, D. (2010): La paupérisation des Français. Paris: Armand Colin.

CunHA, A. (2007): Transformations urbaines et urbanisme durable. - In: Vues sur la ville 17: 1.

CunHA, A. \& J.-F. Bотн (2004): Métropolisation, villes et agglomérations. Structures et dynamiques sociodémographiques des espaces urbains. - Neuchâtel: Office fédéral de la statistique.

Cunha, A. \& O. Schmid (2007a): Exclusion urbaine et logement: de l'urgence au système «D». - Lausanne: Institut de Géographie.

Cunha, A. \& O. Schmid (2007b): Sans domicile fixe: figures, trajectoires et politiques. - Lausanne: Institut de Géographie.

Cunha, A., Bochet, B., Both, J.-F., Mager, C. \& L. VuAGNIAUX (2007): Étalement urbain, mobilité résidentielle et aspirations des ménages. L'agglomération lausannoise. - Lausanne: Observatoire de la ville et $\mathrm{du}$ développement durable.

Cunha, A., Huissoud, T., Stofer, S. \& M. Schuler (2003): Structures et tendances de la différenciation dans les espaces urbains en Suisse. - In: WiCKER, H.R., Fibbi, R. \& W. Haug (éds): Migration und die Schweiz. Ergebnisse des Nationalen Forschungsprogramms «Migration und interkulturelle Beziehungen». - Zürich: Seismo Verlag: 175-196.

Cunha, A., MAger, C. \& O. Schmid (2009): La quadrature du cube. Analyse des besoins et des prestations en matière de prise en charge des personnes dépendantes aux drogues et à l'alcool dans le canton de Fribourg. - Lausanne: Institut de Géographie.

Donzelot, J. (2004): La ville à trois vitesses. Relégation, périurbanisation, gentrification. - In: Esprit 303: 14-39.
Donzelot, J. (2006): Quand la ville se défait. Quelle politique face à la crise des banlieues ? - Paris: Seuil. Donzelot, J. (2009): Vers une citoyenneté urbaine? La ville et l'égalité des chances. - Paris: Rue d'Ulm Editions.

Fitoussi, J.-P., Laurent, E. \& J. Maurice (2004): Ségrégation urbaine et intégration sociale. - Paris: La Documentation française.

Huissoud, T., Stofer, S., Cunha, A. \& M. Schuler (1999): Structures et tendances de la différenciation dans les espaces urbains en Suisse. - Rapport de recherche IREC 145, Lausanne: École polytechnique fédérale

KuZNETS, S. (1955): Economic growth and income inequality. - In: The American Economic Review 45, 1: 1-28.

LandaIs, C. (2007): Les hauts revenus en France (1998-2006). Une explosion des inégalités? - Paris: Paris School of Economics.

Mager, C. \& L. Matthey (2010): Sages comme des images? Le leurre du marketing urbain. - In: Tracés 10: $10-12$

MAGeR, C. \& S. Stofer (2009): Insertion professionnelle et marchés transitoires. Quelles pratiques en Suisse et en Europe? - Sion: Service de l'Action sociale, État du Valais.

Matthey, L. \& B. Steiner (2009a): Des identités à la mesure du Monde? Mondialité, singularité et altérité au spectre des associations de migrants (un exemple suisse). - In: Lieux communs 12:31-44.

Matthey, L. \& B. Steiner (2009b): Nous, moi - les autres. Les associations de migrants et la formation de l'identité: une approche internaliste. - Berne-Wabern: Commission fédérale pour les questions de migration. Meyer, J.-B. (2008): La circulation des compétences, un enjeu pour le développement. - In: Annuaire suisse de politique de développement 27,2: 53-67.

Nisbet, R. (1959): The decline and fall of social class. - In: Pacific Sociological Review 2, 1: 119-129.

OfFICE FÉdÉRAL DE LA STATISTIQUe - OFS (2006): L'enquête suisse sur la structures des salaires 2004. - Neuchâtel: OFS.

OfFICE FÉDÉRAL DE LA STATISTIQUE - OFS (2008): Indicateur de pauvreté pour la compensation des charges au titre des facteurs socio-démographiques dans le cadre de la RPT. - Neuchâtel: OFS.

OfFICE FÉdÉRAL DE LA STATISTIQUE - OFS (2009a): La pauvreté des personnes en âge de travailler.- Neuchâtel: OFS.

OFFICE FÉDÉRAL DE LA STATISTIQUE - OFS (2009b): Statistiques de l'aide sociale. Résultats nationaux. - Neuchâtel: OFS.

Office fédéral de la statistique - OFS (2010): Recensement des entreprises 2008. Les principaux résultats en bref. - Neuchâtel: OFS.

ORganisation DE COOPÉRATION ET DE DÉvelopPEMENT ÉCONOMiQues - OCDE (2004): Réglementation rela- 
tive à la protection de l'emploi et performance du marché du travail. - In: Perspectives de l'emploi: 65138. - Paris: OCDE.

PAKulski, J. \& M.J. WATERs (1996): The reshaping and dissolution of social class in advanced society. - In: Theory and Society 25: 667-691.

Paugam, S. (1991): La disqualification sociale. - Paris: Presses Universitaires de France.

Paugam, S. \& N. Duvoux (2008): La régulation des pauvres. - Paris: Presses Universitaires de France.

Paugam, S. \& C. Martin (2009): La nouvelle figure du travailleur précaire assisté. - In: Lien social et politiques 61: 13-19.

Racine, J.-B. \& V. Noseda (2005): Violences urbaines. Une exploration au-delà des interprétations reçues. - Lausanne: Travaux et recherches de l'Institut de Géographie 29.

Racine, J.-B., Marengo, M., \& C.-A. Blanc (2004): De l'État Providence à la solidarité communautaire. Le monde associatif à Lausanne (Agenda 21). Vers un nouveau projet de société locale. - Lausanne: Travaux et recherches de l'Institut de Géographie 30.

Rérat, P., Piguet, E., Besson, R. \& O. Söderström (2008): Les âges de la ville. Mobilité résidentielle, parcours de vie et attractivité des villes suisses. - In: Geographica Helvetica 63, 4: 261-271.

Rosanvallon, P. (1981): La crise de l'État-providence. - Paris: Seuil.

RosenAU, J.N. \& J.P. SINGH (2002): Information technologies and global politics. The changing scope of power and governance. - Albany: State University of New York Press.

SEN, A. (2000): Repenser l'inégalité. - Paris: Seuil.

Simmel, G. (1908 [2005]): Les pauvres. - Paris: Presses Universitaires de France.

TAskin, L. \& M. Schots (2005): Flexibilité du temps de travail et relation d'emploi. - In: Économies et Sociétés 39, 8: 1471-1501.

Thisse, J.-F. (2004): Villes et économie. - Paris: La Documentation française.

ZhaO, B., Ondrich, J. \& J. Yingerm (2006): Why do real estate brokers continue to discriminate? Evidence from the 2000 Housing Discrimination Study. - In: Journal of Urban Economics 59: 394-419.

\footnotetext{
Résumé: Les villes au piège de la précarité. Généalogie d'un souci des usages fragiles de l'espace urbain Cette contribution questionne la montée de la précarité dans les centres urbains en Suisse. Elaborant une perspective réflexive et critique, elle détaille tout d'abord les mécanismes économiques, institutionnels et les effets de lieux qui la fondent. Dans un deuxième temps, ce sont les modalités émergentes d'accompagnement des usages fragiles de la ville qui sont explicitées. Deux types de sources sont mobilisées: des données et conclusions tirées de la littérature spécifique;
}

des données d'enquêtes réalisées depuis 2004 à l'Observatoire de la ville et du développement durable de l'Université de Lausanne.

Mots-clés: géographie critique, fragilités urbaines, précarité, Suisse

\section{Abstract: Cities ensnared in precarity. A genealogy of concern about urban fragility}

This article examines the spread of urban precarity in Switzerland. Taking a reflexive and critical perspective, it begins by describing current economical and institutional mechanisms as well as the underlying geographical data. This is followed by an overview of the political measures encouraging use of urban facilities by persons in difficult contexts. The article makes use of two types of sources: data and conclusions drawn from a review of specialized literature, as well as results of survey data collected since 2004 at the Observatory of the City and Sustainable Development (University of Lausanne).

Keywords: critical geography, urban fragility, precarity, Switzerland

\section{Zusammenfassung: Städte in der Falle der Prekarität Genealogie des Bemühens um die fragile Nutzung des städtischen Raums}

Dieser Beitrag setzt sich mit der Frage der Zunahme der Prekarität in den städtischen Zentren der Schweiz auseinander. Mit einer reflexiven und kritischen Perspektive beschreibt er zunächst die wirtschaftlichen und institutionellen Mechanismen sowie die ihnen zugrunde liegenden geographischen Daten. Anschliessend werden die politischen Begleitmassnahmen der Nutzung der Stadt durch Personen in prekärer Lage vorgestellt. Der Beitrag stützt sich auf zwei Arten von Quellen: Daten und Schlussfolgerungen, die aus der spezifischen Literatur entommen sind sowie Ergebnisse von Studien, die seit 2004 am Observatoire de la ville et du développement durable der Universität Lausanne durchgeführt wurden.

Schlüsselwörter: Kritische Geographie, Prekarität in den Städten, Schweiz

Dr. Christophe Mager, Institut de Géographie, Université de Lausanne, CH-1015 Lausanne, Suisse. e-mail: Christophe.Mager@unil.ch

Dr. Laurent Matthey, Fondation Braillard Architectes, 16, rue Saint-Léger, CH-1205 Genève, Suisse. e-mail: Laurent.Matthey@braillard.ch

\section{Manuskripteingang/received/manuscrit reçu le 30.6.2010}

Annahme zum Druck/accepted for publication/accepté pour publication: 5.12 .2010 